\title{
Sepsis, una tarea de todos
}

\author{
CARLOS ACUÑA A. ${ }^{1}$ \\ 1. Jefe UCI Pediátrica. Hospital Luis Calvo Mackenna. \\ Universidad de Chile. Departamento de Pediatria y Cirugía Infantil Oriente.
}

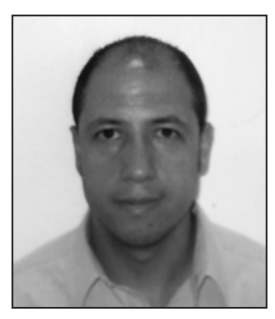

La sepsis, como otros aspectos de la medicina, no está ajena a los cambios de la sociedad actual. Actualmente el énfasis está puesto en un enfoque global, aunque sin dejar de lado importantes avances en la comprensión de la fisiología y alternativas terapéuticas.

Nos encontramos dentro de un ambiente favorable para desarrollar a nivel mundial y local estrategias para mejorar el manejo/pronóstico de la sepsis en pediatría. La sepsis, a pesar de su notable incidencia, es una entidad prácticamente desconocida para la población general. A nivel mundial es una de las causas más frecuentes de mortalidad, siendo la principal causa de muerte por infección a pesar de los avances en la medicina de las últimas décadas, incluidas las vacunas, los antibióticos y la atención de urgencia/unidad de cuidados intensivos (UCI), con tasas de mortalidad hospitalaria entre 30 y $60 \%$.

Haciéndose cargo de este problema, varias iniciativas globales se encuentran actualmente en curso. La campaña de supervivencia a la sepsis (Surviving Sepsis Campaign), iniciada el 2002 planteó como objetivo reducir su mortalidad en un $25 \%$ a 5 años plazo, a través de una agenda de 7 puntos, que incluían la sensibilización hacia la sepsis, mejoría en el diagnóstico, incremento de la utilización de un tratamiento adecuado, la educación de los profesionales sanitarios, mejorar la atención post-UCI, elaboración de directrices de atención y la implementación de un programa de mejora continua .
La Federación Mundial de Cuidados Intensivos Pediátricos y las Sociedades de Cuidados Críticos (WFPICCS) anunciaron en el Congreso Mundial en Ginebra, Suiza 2007, su intención de lanzar una primera iniciativa de salud global, que consistía en un programa de mejora de la calidad a través de la realización de intervenciones simples para mejorar la calidad de atención a los niños con sepsis.

Como parte de esta estrategia recientemente el 15 de Septiembre celebramos el segundo Día Mundial de la Sepsis (World Sepsis Day), iniciativa que busca posicionar y alertar sobre la sepsis a nivel global.

Esta es una de las pocas condiciones que puede afectar por igual a la población con escasos recursos y aquellas con mejores ingresos. En el mundo desarrollado, la sepsis ha aumentando dramáticamente a un ritmo anual de $8-13 \%$ en la última década, con una mortalidad mayor que el cáncer de colon y de mama, juntos. Las razones son múltiples, influyendo el envejecimiento de la población, el aumento del uso de intervenciones de alto riesgo en todos los grupos de edad, el desarrollo de gérmenes resistentes a los antimicrobianos e infecciones más virulentas. Por otra parte, en el mundo en desarrollo, la desnutrición, la pobreza, la falta de acceso a las vacunas y el tratamiento oportuno, son los factores determinantes en el pronóstico de la sepsis. Esto se refleja por ejemplo en un estudio realizado en nuestra unidad de cuidados intensivos, donde analizamos los úl-

Recibido el 22 de noviembre de 2013.

Correspondencia a:

Carlos Acuña A.

E-mail: cacuna@calvomackenna.cl 
timos 20 años destacando como diagnóstico de ingreso en 1991 la desnutrición con un 43,8\% versus un $6,9 \%$ en el 2011 y un descenso en la mortalidad global desde un $15,3 \%$ a un $3 \%$.

El Día Mundial de la Sepsis se plantean 5 objetivos como meta para el año 2020, a saber: reducción de la incidencia de la sepsis al menos en un $20 \%$ mediante estrategias de prevención, mejora de la supervivencia de niños y adultos en todo el mundo, sensibilización pública y profesional, asegurar un mejor acceso a los servicios de rehabilitación adecuados y la creación y mantenimiento de una base de datos de incidencia y resultados.

Al enfrentarnos a la problemática de la sepsis surgen una serie de interrogantes: ¿Cuál es nuestro rol en el contexto de iniciativas mundiales/globales? ¿Cómo se encuentra nuestro país en este plano? ¿Qué estrategias tenemos a nivel ministerial? ¿Por qué tiene menor cobertura que otras patologías que tienen una marcada menor morbi/mortalidad?

Una clara estrategia a seguir es la de promover y educar, donde artículos como los desarrollados por el Donoso y cols en este número de la Revista Chilena de Pediatría, con una excelente actualización de la fisiopatología y terapéutica de esta enfermedad, apuntan en la dirección adecuada.

El entrenamiento y la protocolización del manejo de estos pacientes ha demostrado una reducción importante en la mortalidad, sin embargo, trabajos como el de Paul R. y cols ${ }^{1}$ sobre adherencia a las metas de reanimación según PALS nos muestra que el cumplimiento en relación a la reanimación con fluidos es de un $37 \%$, inotropos $35 \%$, adherencia al bundle completo sólo $19 \%$, con un impacto en la estadía hospitalaria donde aquellos que seguían las guías tanto en lo relacionado con la reanimación con fluidos como a la adherencia al algoritmo total presentaban una estadía un 57\% menor comparados con aquellos que no cumplían el algoritmo PALS. Por otra parte, Launay E. y cols ${ }^{2}$, analiza el manejo sub óptimo de los pacientes fallecidos por sepsis, destacando 3 niveles de errores: diagnóstico, tratamiento y comunicación.

El llamado entonces es a adherir a los enunciados del día mundial de la sepsis mejorando el futuro de nuestros niños.

\section{Referencias}

1.- $\quad$ Paul R, Neuman MI, Monuteaux MC, Melendez E: Adherence to PALS Sepsis Guidelines and Hospital Length of Stay. Pediatrics 2012; 130 (2).

2.- Launay E, Gras-Le Guen C, Martinot A, et al: Suboptimal care in the initial management of children who died from severe bacterial infection: a population-based confidential inquiry. Pediatr Crit Care Med 2010; 11 (4): 469-74. 